\title{
SAÚDE BUCAL DE PACIENTES COM TRANSTORNOS ALIMENTARES: O MARCANTE PAPEL DO CIRURGIÃO-DENTISTA.
}

\section{ORAL HEALTH OF PATIENTS WITH EATING DISORDERS: THE ROLE OF THE DENTIST}

\author{
Vivian Narana Ribeiro El Achkar* \\ Graziella Nuermberg Back-Brito** \\ Cristiane Yumi Koga-Ito**
}

\begin{abstract}
RESUMO
A Anorexia Nervosa e Bulimia Nervosa são transtornos alimentares de ordem comportamental podendo ser diagnosticadas primeiramente pelo cirurgião-dentista por apresentarem efeitos marcantes sobre a saúde bucal. A falta de familiaridade de profissionais de saúde com o assunto tem dificultado o diagnóstico, atrasando o tratamento e, consequentemente, aumentado o risco de complicações clínicas desses transtornos. Considerando-se a crescente incidência dessas síndromes, associada à alta morbidade, preconiza-se o melhor conhecimento de suas manifestações clínicas, bem como de complicações associadas para que o diagnóstico possa ser realizado mais precocemente, evitando-se, assim, que os pacientes cheguem para o tratamento apenas quando seu estado já esteja grave. A morte de uma modelo brasileira e de uma estudante de moda no final do ano de 2006 teve repercussão mundial e reacendeu o interesse pelo tema. O objetivo deste artigo é alertar os cirurgiõesdentistas sobre seu papel-chave na prevenção das consequências bucais dos transtornos alimentares, por meio de uma discussão de base diagnóstica e terapêutica. É essencial que o cirurgião-dentista tenha segurança no diagnóstico, já que muitas vezes é o primeiro profissional de saúde a detectá-los, permitindo manejo e encaminhamento adequado e promovendo saúde bucal e geral desses pacientes.
\end{abstract}

DESCRITORES: Anorexia nervosa • Bulimia nervosa • Saúde bucal • Transtornos da alimentação

\section{ABSTRACT}

Anorexia Nervosa and Bulimia Nervosa are eating disorders and can be first diagnosed by the dentist, due to marked effects on oral health. Lack of knowledge of health professionals on this issue has hampered the diagnosis, delaying treatment and, consequently, increased the risk of clinical complications. The increasing incidence of these syndromes, associated with high morbidity, emphasizes a better understanding of its clinical manifestations, and complications related to the diagnosis. The early diagnosis can prevent patients for looking for treatment just when their condition is already severe. The death of a Brazilian model and a fashion student at the end of 2006 had worldwide repercussions and rekindled interest in the topic. The aim of this paper is to alert dentists on their role in the prevention of oral consequences of eating disorders through a discussion of basic diagnostic and therapeutic. It is essential that the dentist can make safety and early diagnosis, as frequently they are the first health professionals to detect them. This can allow the better management and appropriate referral and promoting oral and general health of these patients.

DESCRIPTORS: Anorexia nervosa • Bulimia nervosa $\bullet$ Oral health • Eating disorders

\footnotetext{
* Graduanda da Faculdade de Odontologia de São José dos Campos - Universidade Estadual Paulista (UNESP) - SP - Brasil. Email: vivian.narana@terra.com. br

** Doutora - Departamento de Biociências e Diagnóstico Bucal, Faculdade de Odontologia de São José dos Campos - Universidade Estadual Paulista (UNESP) - SP - Brasil. Email: grazinback@yahoo.com.br

*** Professora Adjunta - Departamento de Biociências e Diagnóstico Bucal, Faculdade de Odontologia de São José dos Campos - Universidade Estadual Paulista (UNESP) - SP - Brasil. Email: cristiane@fosjc.unesp.br
} 
ACHKAR VNRE

$B A C K-B R I T O \quad G N$

KOGA-ITO CY

SAÚDE BUCAL DE PACIENTES COM

TRANSTORNOS

ALIMENTARES : 0

MARCANTE PAPEL

DO CIRURGIÃO-

DENTISTA .
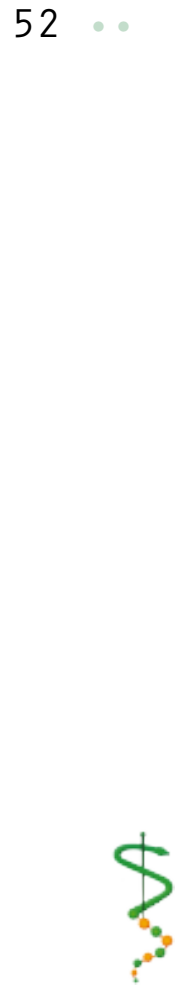

REV, ODONTOL.

UNIV, CID, SÃO

PAULO

2012; $24(1): 51-6$,

$J A N-A B R$

\section{N T RO DUÇ ÃO}

O número de casos de transtornos alimentares vem aumentando significativamente ao longo dos anos, estimulado, sobretudo por uma sociedade moderna que elege a magreza como símbolo de sucesso e beleza. Nos últimos anos a valorização excessiva da forma e do peso do corpo tem levado muitas pessoas, principalmente mulheres, a verdadeiros sacrifícios, que podem comprometer a saúde, como dietas radicais e exercícios físicos em excesso, com o intuito de conseguirem chegar ao corpo ideal (Segal et al. $\left.{ }^{1}, 1995\right)$.

Os relatos iniciais de um padecimento autoimposto por meio de restrição alimentar datam da Idade Média e estavam relacionados a uma conduta religiosa de privação (Abuchaim², 2002, Cordás e Claudino $^{3}$, 2002, Cordás $\left.{ }^{4}, 2004\right)$. O termo anorexia deriva do grego "an" deficiência ou ausência de, e "orexis", apetite. A Anorexia Nervosa é caracterizada pela perda de peso à custa de dieta extremamente restrita, busca desenfreada pela magreza, distorção da imagem corporal e alterações do ciclo menstrual (Cordás ${ }^{4}, 2004$, Borges et al. $\left.{ }^{5}, 2006\right)$. A bulimia nervosa apresenta uma história mais recente, surgiu como uma síndrome na década de 70, relacionada à Anorexia Nervosa. O termo bulimia deriva do grego "bous" boi e "limos" fome, designando um apetite de comer um boi. É caracterizada em sua forma típica pela ingestão compulsiva e rápida de grande quantidade de alimento, com pouco ou nenhum prazer, alternada com comportamento dirigido para evitar ganho de peso e medo mórbido de engordar (Abuchaim $^{2}$, 2002, Cordás e Claudino ${ }^{3}$, 2002, Cordás ${ }^{4}, 2004$, Borges et al. , 2006, Cordás e Segal $\left.{ }^{6}, 1995\right)$.

Embora classificados separadamente no DSM-IV (Diagnostic and Statistical Manual IV edition) e CID-10, os dois transtornos acham-se intimamente relacionados por apresentarem psicopatologia comum: uma ideia prevalente envolvendo a preocupação excessiva com o peso e a forma corporal (medo de engordar), que leva as pacientes a se engajarem em dietas extremamente restritivas ou a utilizarem métodos purgativos (Cordás ${ }^{4}, 2004$, Borges et al. ${ }^{5}, 2006$, Romaro e Itokazu $\left.{ }^{7}, 2002\right)$. Os estudos com metodologias e amostras diversas dificultam a obtenção de dados epidemiológicos mais fiéis, mas de uma forma geral, a prevalência de Anorexia Nervosa varia entre $0,5 \%$ e $3,7 \%$ e de Bulimia Nervosa de 1,1\% e 4,2\% da população (Pinzon e Nogueira ${ }^{8}$, 2004). Predominantemente, os transtornos alimentares ocorrem em mulheres jovens, com uma prevalência média de relação entre homem e mulher de 1:10 e até 1:20 de acordo com os critérios utilizados (Klein e Walsh', 2004).

Os transtornos alimentares são acompanhados de várias complicações clínicas relacionadas ao comprometimento do estado nutricional e às práticas compensatórias inadequadas para o controle do peso, como a indução de vômitos ou uso de diuréticos, enemas e laxativos. A morbidade e mortalidade são expressivas, a Anorexia Nervosa apresenta a maior taxa de mortalidade dentre todos os distúrbios psiquiátricos, cerca de 0,56\% ao ano. Esse valor é em torno de 12 vezes maior que a mortalidade das mulheres jovens na população em geral. As principais causas de morte são: complicações cardiovasculares, insuficiência renal e suicídio (Assumpção e Cabral ${ }^{10}$, 2002). A Anorexia e Bulimia Nervosas são descritas como transtornos alimentares e não como doenças, por ainda não se conhecer bem sua etiopatogenia. No entanto, fatores genéticos, sociais, familiares e psicológicos parecem estar relacionados (Klein e Walsh $\left.{ }^{9}, 2004\right)$. Devido à associação desses fatores etiológicos e à presença de inúmeras complicações clínicas, o tratamento desses transtornos requer uma equipe multidisciplinar com psiquiatra, endocrinologista, ginecologista, pediatra, terapeutas individual e familiar, nutricionista, enfermagem e outros profissionais da área da saúde (Segal et al. ${ }^{1}, 1995$, Assumpção e Cabral ${ }^{10}$, 2002, Cordás ${ }^{11}$, 2001). O cirurgião-dentista deve fazer parte do atendimento multidisciplinar e pode ser o primeiro profissional da saúde a detectar os sinais e sintomas que indicam algum dos transtornos alimentares, por meio de observação de algumas manifestações intra e extrabucais. 


\section{REVISÃO DE LITERATURA}

As manifestações extrabucais relacionadas à restrição alimentar estão associadas à má nutrição. Além do baixo peso e aparência pré-puber, a pele apresenta-se pálida ou com coloração amarelada, seca, sem brilho e por vezes coberta por uma fina camada de pelos (lanugo); os cabelos são ralos, finos e opacos e unhas quebradiças (Alonso et al. ${ }^{12}, 2001$, DeBate et al. ${ }^{13}$, 2005, Caldeira et al. ${ }^{14}$, 2000). Essas características podem não estar presentes em pacientes bulímicos, pois, na maioria das vezes, estes mantêm um peso normal (Woodmansey ${ }^{15}, 2000$, Seabra et al. $\left.{ }^{16}, 2004\right)$. A indução de vômitos pode ocasionar um aumento de volume das glândulas salivares, frequentemente das glândulas parótidas, denominadas sialoadenose e sialose (Alonso et al. ${ }^{12}, 2001$, DeBate et al. ${ }^{13}, 2005$, Coleman et al. ${ }^{17}$, 1998, Touger-Decker $\left.{ }^{18}, 2006\right)$. A deformidade facial mostra-se nítida, ocasionando um rosto inchado e mandíbula quadrada que podem ser observados em $37 \%$ dos anoréxicos e em 53\% dos bulímicos. Sob palpação, a glândula se apresenta macia e geralmente não há relato de dor (Alonso et al. $\left.{ }^{12}, 2001\right)$. Escoriações dorsais da pele das mãos ou calo no dedo utilizado para estimular o reflexo na garganta podem ser observados e são conhecidos como "Sinal de Russel" (Segal et al. ${ }^{1}, 1995$, Assumpção e Cabral ${ }^{10}$, 2002). O vômito provocado é o método mais utilizado por pacientes anoréxicos e bulímicos para prevenir o ganho de peso, correspondendo a $80-90 \%$ dos casos: é um dos processos mais destrutivos que afetam os tecidos dentais duros da cavidade bucal (Caldeira et al. ${ }^{14}, 2000$ ). Essa manifestação é a primeira na qual o cirurgião-dentista poderá realizar um diagnóstico diferencial e detectar um caso de desordem alimentar (de Moor ${ }^{19}, 2004$ ). A erosão é um processo no qual o tecido dental é removido por um processo químico. A regurgitação crônica de conteúdo do ácido estomacal leva com frequência a uma distribuição típica de erosão dental dentro das arcadas dentárias, correspondendo à trajetória do ácido gástrico. Esse tipo específico de lesão de esmalte é denominado de perimólise e ocorre pre- dominantemente nas superfícies palatinas dos anteriores superiores, linguais dos anteriores inferiores e oclusais e linguais dos posteriores (Alonso et al. ${ }^{12}, 2001$, Caldeira et al. ${ }^{14}, 2000$, de Moor $^{19}$, 2004, Pegoraro et al. ${ }^{20}, 2000$, Traebert e Moreira ${ }^{21}$, 2001). As lesões são lisas, com contornos arredondados, sem sinais de pigmentação (Pegoraro et al. $\left.{ }^{20}, 2000\right)$.

Devido à perda de substâncias dentárias, o paciente pode apresentar bordas incisais finas ou fraturadas e as bordas de restaurações sobressaem das superfícies dentais, apresentando um aspecto de ilha. Em casos severos há encurtamento da coroa clínica, devido aos desgastes oclusais de dentes posteriores ocasionando perda de dimensão vertical (Caldeira et al. ${ }^{14}$, 2000, Seabra et al. ${ }^{16}$, 2004). A abrasão mecânica pela escovação logo após o vômito pode agravar o desgaste dental (Alonso et al. ${ }^{12}$, 2001). Geralmente, a erosão dental e consequente exposição da dentina serão acompanhadas de dor. A hipersensibilidade dentinária e o comprometimento estético são os principais fatores que levam os pacientes a procurar por tratamento odontológico (Caldeira et al. $\left.{ }^{14}, 2000\right)$.

Estudos sobre a prevalência de cárie dental em pacientes com transtornos alimentares são controversos. Alguns estudos demonstraram que o risco de cárie é variável, assim como na população em geral (Alonso et al. ${ }^{12}$, 2001, de Moor ${ }^{19}, 2004$, Milosevic e Slade ${ }^{22}$, 1989). Para alguns pesquisadores, a ingestão de carboidratos e açúcares pelos pacientes bulímicos nos episódios de compulsão alimentar favorece o desenvolvimento de cárie (Seabra et al. ${ }^{16}, 2004$, Traebert e Moreira ${ }^{21}, 2001$ ). Portanto, a diferença nos índices de cáries de pacientes com transtornos alimentares pode ser atribuída à higiene bucal, cariogenicidade da dieta e uso de medicamentos que ocasionam xerostomia, sendo que a associação de frequência de vômitos com incidência de cárie ainda é incerta (de Moor $^{19}$, 2004). A xerostomia nesses pacientes pode ser resultante tanto das práticas purgativas, quanto dos medicamentos utilizados no tratamento dos transtornos. A indução de vômito, uso de laxantes e diuréticos causam um decréscimo no volume total de fluido, diminuin-
ACHKAR VNRE

BACK-BRITO GN

KOGA-ITO CY

SAÚdE BUCAL DE

PACIENTES COM

TRANSTORNOS

ALIMENTARES : O MARCANTE PAPEL DO CIRURGIÃO DENTISTA.

\section{3}

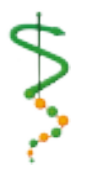

REV, ODONTOL.

UNIV, CID, SÃo

PAULO

2012; $24(1): 51-6$ JAN - ABR 
ACHKAR VNRE $B A C K-B R I T O \quad G N$

KOGA-ITO CY

SAÚDE BUCAL DE PACIENTES COM

TRANSTORNOS

ALIMENTARES : 0

MARCANTE PAPEL DO CIRURGIÃODENTISTA .

54

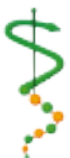

REV, ODONTOL.

UNIV, CID, SÃO

PAULO

2012; 24(1): $51-6$,

$J A N-A B R$
I SSN 1983-5183

do, também, o fluxo salivar. A administração de antidepressivos, anticolinérgicos e tranquilizantes no tratamento, pode, igualmente, induzir xerostomia. A xerostomia apresentada em alguns pacientes anoréxicos e bulímicos pode também reduzir a capacidade tampão e o pH salivar (Alonso et al. ${ }^{12}, 2001$, de Moor ${ }^{19}$, 2004).

Como os pacientes acometidos por transtornos alimentares são geralmente jovens, a doença periodontal é raramente diagnosticada (de Moor ${ }^{19}$, 2004). Gengivite, papilas interdentais hipertrofiadas devido à irritação constante do vômito ácido e a ressecção gengival e abrasão por escovação podem ser encontradas (Alonso et al. ${ }^{12}$, 2001, Seabra et al. ${ }^{16}, 2004$, de Moor $^{19}$, 2004). O trauma da mucosa bucal e da faringe pode ser observado em pacientes com compulsão alimentar e naqueles que induzem o vômito. Esse trauma pode ser causado tanto pela rápida ingestão dos alimentos quanto pela força na regurgitação. $O$ palato mole pode apresentar-se ulcerado devido a objetos utilizados para provocar o vômito, como dedos, pentes, escovas de dentes (Alonso et al. ${ }^{12}, 2001$, Seabra et al. ${ }^{16}, 2004$, de Moor $^{19}$, 2004).

Outras alterações que podem ocorrer são queilite angular, candidoses e herpes, como consequência da toxidade do vômito (Alonso et al. ${ }^{12}, 2001$, Milosevic e Slade ${ }^{22}$, 1989). Além da importância do conhecimento desses sinais e sintomas visando o diagnóstico, o cirurgião-dentista tem um papel fundamental no que concerne à prevenção e manutenção da saúde bucal desses pacientes. Na suspeita da presença de um desses transtornos, o cirurgião-dentista deve abordar o assunto de forma que se obtenha a confiança do paciente, inquirindo sobre seus hábitos alimentares e a possibilidade de existência de problemas gastrointestinais e instruí-lo sobre a necessidade de avaliação médica (Traebert e Moreira ${ }^{21}, 2001$ ).

Ao confirmar o diagnóstico de Anorexia Nervosa ou Bulimia Nervosa, o profissional deve informar o paciente sobre as causas e consequências dessas patologias da cavidade bucal (Cordás $\left.{ }^{11}, 2001\right)$. Segundo Burke et al. ${ }^{23}$ (1996), o manejo do paciente anoréxico ou bulímico em rela- ção à saúde bucal deve envolver cuidados emergenciais, preventivos e restauradores. Dentre os cuidados emergenciais, encontra-se o alívio da dor e melhora da estética. Como alívio da dor, propõe-se a proteção da dentina exposta, com a utilização de cimentos de ionômero de vidro, vernizes fluoretados ou, se necessário, tratamento endodôntico (Seabra et al. ${ }^{16}$, 2004, Traebert e Moreira ${ }^{21}$, 2001). O tratamento restaurador, nesse momento, objetiva a melhora da estética e deve ser realizado para auxiliar no tratamento psicológico e na recuperação da autoestima do paciente (Seabra et al. ${ }^{16}, 2004$, Traebert e Moreira ${ }^{21}$, 2001).

A prevenção na área de saúde bucal, nesses pacientes, abrange diversas medidas. Para pacientes que induzem o vômito, deve-se salientar os riscos decorrentes de tal prática para cavidade bucal. O cirurgião-dentista deve orientar o paciente a não escovar os dentes imediatamente após a indução de vômito e prescrever bochechos com fluoreto de sódio a 0,05\% ou bochechos com água e bicarbonato de sódio para neutralizar o $\mathrm{pH}$ do ambiente bucal. Deve-se, ainda, indicar a utilização de cremes dentais com alta concentração de flúor e baixa abrasividade, além do uso de escovas extramacias (Alonso et al. ${ }^{12}$, 2001, Seabra et al. ${ }^{16}, 2004$, Touger-Deck$\mathrm{er}^{18}, 2006$, Traebert e Moreira $\left.{ }^{21}, 2001\right)$. A aplicação tópica de flúor no consultório odontológico também contribui na prevenção de erosão dental e promove remineralização do esmalte dental (Alonso et al. $\left.{ }^{12}, 2001\right)$

O aconselhamento dietético deve ser realizado no sentido de evitar alimentos e bebidas ácidas, como refrigerantes, sucos e frutas cítricas (Touger-Decker ${ }^{18}, 2006$, Traebert e Moreira ${ }^{21}$, 2001). Pacientes com episódios bulímicos de compulsão alimentar devem ser orientados sobre o ataque cariogênico ao qual estão suscetíveis devido à ingestão de grandes quantidades de carboidratos e açúcares (Traebert e Moreira $^{21}$, 2001). No caso de xerostomia, o cirurgião-dentista pode indicar o uso de chicletes sem açúcar para estimular a produção de saliva e, nos casos mais severos de xerostomia, a utilização de saliva artificial (Alonso et al.12, 2001). 
O tratamento restaurador definitivo só deve ser efetuado quando o paciente encontra-se psicologicamente estabilizado (Alonso et al. ${ }^{12}, 2001$ ). Para o plano de tratamento deve-se levar em consideração o comprometimento das estruturas dentais, podendo variar de um procedimento simples de restauração até reabilitações bucais complexas em pacientes com perda da dimensão vertical (Seabra et al. ${ }^{16}$, 2004, Traebert e Moreira ${ }^{21}, 2001$, Little ${ }^{24}$, 2002).

\section{CONCLUSÃO}

A má nutrição e a prática de indução de vômito decorrentes da Anorexia Nervosa e da Bulimia Nervosa podem ocasionar inúmeras alterações sistêmicas e bucais. O cirurgião-dentista é, muitas vezes, o primeiro profissional de saúde a detectá-las e tem um papel fundamental no diagnóstico precoce desses transtornos alimentares, na prevenção e promoção de saúde bucal em tais pacientes.

\section{REFERÊNCIAS}

1. Segal A, Cordás TA, D'Elia FLGM, Larino MA, Alvarenga M, Bucaretchi $\mathrm{H}$, et al. Bulimia nervosa II: tratamento J bras psiquiatr 1995 out.;44(supl.1):S25-S31.

2. Abuchaim ALG. Aspectos históricos da anorexia e bulimia nervosas Rev psiquiatr Rio Gd Sul 2002 maio-ago;24(2):199-208.

3. Cordás TA, Claudino AdM. Transtornos alimentares: fundamentos históricos. Rev Bras Psiquiatr 2002 Dec.;24(suppl.3):03-6.

4. Cordás TA. Transtornos alimentares: classificação e diagnóstico. Rev psiquiatr clín 2004 31(4):154-7.

5. Borges NJBG, Sicchieri JMF, Ribeiro RPP, Marchini JS, Dos Santos JE. Transtornos alimentares: quadro clínico. Medicina (Ribeirão Preto) 2006 jul.-set.;39(3):340-8.

6. Cordás T, Segal A. Bulimia I: aspectos clínicos. J Bras Psiquiatr 1995 44(supl.1):20-4.

7. Romaro RA, Itokazu FM. Bulimia nervosa: revisão da literatura. Psicol Reflex Crit 2002 15(2):407-12.

8. Pinzon V, Nogueira FC. Epidemiologia, curso e evolução dos transtornos alimentares. Rev psiquiatr clín 2004 31(4):158-60.

9. Klein DA, Walsh BT. Eating disorders: clinical features and pathophysiology. Physiol Behav 2004 Apr;81(2):359-74.

10. Assumpção CLd, Cabral MD. Complicações clínicas da anorexia nervosa e bulimia nervosa. Rev Bras Psiquiatr 2002 Dec.;24(suppl.3):29-33.

11. Cordás TA. Transtornos alimentares em discussão. Rev Bras Psiquiatr 2001 Dec.;23(4):178-9.

12. Alonso C, Sabás M, Castillo MA, Weisstaub G, Pascual DM. Trastornos de la conducta alimentaria: repercusiones a nivel oral Rev Asoc Odontol Argent 2001 jul.-ago;89(4):390-5.

13. DeBate RD, Tedesco LA, Kerschbaum WE. Knowledge of oral and physical manifestations of anorexia and bulimia nervosa among dentists and dental hygienists. J Dent Educ 2005 Mar;69(3):346-54.

14. Caldeira TH, Nápole RCD, Busse SR. Erosão dental e a contribuição do cirurgião-dentista no diagnóstico de bulimia nervosa Rev Assoc Paul Cir Dent 2000 nov.-dez.;54(6):465-7. 
ACHKAR VNRE $B A C K-B R I T O \quad G N$

KOGA - ITO CY

SAÚDE BUCAL DE PACIENTES COM TRANSTORNOS ALIMENTARES : 0 MARCANTE PAPEL DO CIRURGIÃODENTISTA .

15. Woodmansey KF. Recognition of bulimia nervosa in dental patients: implications for dental care providers. Gen Dent 2000 Jan-Feb;48(1):48-52.

16. Seabra BGM, Seabra FRG, Almeida RQ, Ferreira JMS. Anorexia nervosa e bulimia nervosa e seus efeitos sobre a saúde bucal. Rev bras patol oral 2004 out.-dez.;3(4):195-8.

17. Coleman H, Altini M, Nayler S, Richards A. Sialodenosis: a presenting sign in bulimia. Head \& Neck 1998 1(2):758-62.

18. Touger-Decker R. Eating disorders: detection and referral--the role of the dental professional. Quintessence Int 2006 Mar;37(3):199-201.

19. de Moor RJ. Eating disorder-induced dental complications: a case report. J Oral Rehabil 2004 Jul;31(7):725-32.

20. Pegoraro CN, Sakamoto FFO, Domingues LA. Perimólise: etiologia, diagnóstico e prevenção Rev Assoc Paul Cir Dent 2000 mar.-abr.;54(2):156-61.

21. Traebert J, Moreira E. Transtornos alimentares de ordem comportamental e seus efeitos sobre a saúde bucal na adolescência. Pesqui Odontol Bras 2001 Oct-Dec.; 15(4):359-63.

22. Milosevic A, Slade PD. The orodental status of anorexics and bulimics. Br Dent J 1989 Jul 22;167(2):66-70.

23. Burke FJ, Bell TJ, Ismail N, Hartley P. Bulimia: implications for the practising dentist. Br Dent J 1996 Jun 8;180(11):421-6.

24. Little JW. Eating disorders: dental implications. Oral Surg Oral Med Oral Pathol Oral Radiol Endod 2002 93(2):138-43.

Recebido em: 14/02/2011

Aceito em: 28/03/2011 